\title{
Análise Volumétrica Tardia após Implante de Stents Eluidores de Sirolimus versus Biolimus A9
}

\author{
Eduardo Missel ${ }^{1}$, Alexandre Abizaid ${ }^{1}$, Felipe Cesar ${ }^{1}$, Marinella P. Centemero', Luiz A. Mattos ${ }^{1}$,

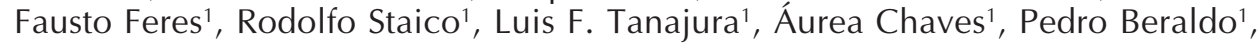 \\ Andrea Abizaid', Amanda Sousa', Eberhard Grube², J. Eduardo Sousa ${ }^{1}$
}

\section{RESUMO}

Introdução: Os stents eluidores de sirolimus (SES) reduzem significativamente a incidência de reestenose e de eventos cardíacos maiores em comparação a stents convencionais. O biolimus A9 (BA9), um análogo do sirolimus, demonstrou eficácia e segurança similares no estudo randomizado e controlado STEALTH I. O objetivo deste estudo é comparar a eficácia do stent eluidor de BA9 com os SES, assim como seu desempenho em relação aos respectivos grupos controle, utilizando a análise volumétrica pelo ultra-som intracoronário (3D-USIC). Método: No total, 45 pacientes foram randomizados 2:1 para o grupo submetido a implante de stents eluidores de BA9 $(n=30)$ ou para o grupo controle $(n=15)$. Os resultados de angiografia coronária quantitativa e 3D-USIC foram comparados a uma série histórica de pacientes submetidos a implante de SES $(n=30)$ ou controles $(n=15)$. As características clínicas e angiográficas foram semelhantes entre os grupos, exceto pela maior quantidade de lesões tipo $\mathrm{C}$ e mulheres no grupo dos stents eluidores de BA9. Resultados: Aos seis meses de seguimento, a perda tardia intrastent foi significativamente inferior nos grupos de BA9 e SES em comparação aos respectivos controles, mas foi semelhante quando estes foram comparados entre si $(0,24$ $\pm 0,39 \mathrm{~mm}$ vs. $0,15 \pm 0,38 \mathrm{~mm}$, respectivamente; $p=\mathrm{NS}$ ). A obstrução volumétrica intra-stent foi significativamente reduzida nos grupos de BA9 e SES em comparação aos controles, mas semelhante quando comparados entre si $(2,23 \%$ vs. $3,30 \%$, respectivamente; $p=N S)$. Conclusão: Os stents eluidores de BA9 reduziram significativamente a proliferação neo-intimal se comparados ao grupo controle, com eficácia similar à observada para os SES.

DESCRITORES: Ultra-som. Contenedores. Coronariopatia. Materiais revestidos biocompatíveis. Sirolimo, análogos \& derivados.

\section{SUMMARY}

Late Volumetric Analysis After Implantation of

Sirolimus- Versus Biolimus A9-eluting Stents

Introduction: Sirolimus-eluting stents (SES) significantly reduce restenosis and major adverse cardiac events (MACE) compared to bare metal stents (BMS). The novel sirolimus analog, Biolimus A9T (BA9), presented similar safety and efficacy in the randomized, controlled STEALTH I trial. This study compared the efficacy of a BA9-eluting stent versus sirolimus-eluting and bare metal control stents. Methods: Forty-five patients with de novo coronary lesions were randomly assigned in a 2:1 basis to receive either BA9-eluting $(n=30)$ or bare metal $(n=15)$ S-stents. Quantitative coronary angiography (QCA) and intravascular ultrasound (IVUS), at 6 months, were then compared to a matched series of patients who received either sirolimuseluting $(n=30)$ or bare metal $(n=15)$ Bx Velocity stents. Baseline clinical and angiographic characteristics were similar among all groups, except for a significantly higher percentage of females and Class $\mathrm{C}$ lesions in the BA9eluting stent group. Results: At 6 month follow-up, there was no significant difference in clinical outcomes between any of the groups. QCA revealed significantly lower late loss in both drug-eluting stents (DES) groups compared to bare metal controls, but no significant difference between BA9 and SES groups was observed $(0.24 \pm 0.39 \mathrm{~mm}$ vs. $0.15 \pm 0.38 \mathrm{~mm}, p=\mathrm{NS}$ ). Obstruction volume measured by 3D IVUS was significantly reduced in both DES groups compared to bare metal controls, but did not differ between the BA9 and SES groups $(2.23 \%$ vs. $3.30 \%$, BA9 vs. SES, $p=N S$ ). Conclusions: BA9-eluting stents reduce neointimal hyperplasia, safely and effectively, compared to BMS, and the magnitude of this inhibition is similar to that of SES.

DESCRIPTORS: Ultrasonics. Stents. Coronary disease. Coated materials, biocompatible. Sirolimus, analogs \& derivatives.
1 Instituto Dante Pazzanese de Cardiologia - São Paulo, SP.

2 Helios Heart Center - Siegburg, Alemanha.

Correspondência: Alexandre Abizaid. Instituto Dante Pazzanese de Cardiologia. Av. Dr. Dante Pazzanese, 500 - São Paulo, SP CEP 04038-003 - E-mail: aabizaid@uol.com.br Recebido em: 29/1/2008 • Aceito em: 13/2/2008 
0 s stents liberadores de sirolimus são dispositivos altamente eficazes em inibir a proliferação neo-intimal e, conseqüentemente, em reduzir as taxas de reestenose binária e de nova revascularização do vaso tratado ${ }^{1,2}$. Modelos experimentais demonstraram que o biolimus A9 (BA9), um análogo do sirolimus, possui potente efeito antiproliferativo em células musculares lisas, de maneira similar ao sirolimus ${ }^{3}$. O desempenho do BA9 foi testado inicialmente em humanos, no estudo randomizado e controlado Stent Eluting A9 Biolimus Trial in Humans (STEALTH I), revelando marcada redução da perda tardia angiográfica em comparação aos stents convencionais ${ }^{4}$, além de reduzir significativamente o porcentual de obstrução intra-stent ao ultra-som intracoronário ${ }^{5}$. Este estudo tem como objetivo comparar a eficácia desse novo dispositivo com a dos stents eluidores de sirolimus aos seis meses, por meio da análise volumétrica pelo ultra-som intracoronário.

\section{MÉTODO}

De novembro de 2003 a março de 2004, 45 pacientes foram randomizados 2:1 para receber stents eluidores de BA9 $\left(n=30\right.$, BioMatrix ${ }^{\text {TM }}$, Biosensors International, Cingapura) ou stent controle ( $\mathrm{n}=15$, Sstent $^{\mathrm{TM}}$, Biosensors International, Cingapura) e recrutados em nossa instituição para participar do estudo STEALTH I. Esses pacientes foram pareados com dois grupos históricos que receberam stents eluidores de sirolimus $(\mathrm{n}=30)$ (Cypher $^{\mathrm{TM}}$, Cordis Corp., Miami Lakes, FL, Estados Unidos) ou stent controle Bx-Velocity TM $\left.^{\text {TM }}=15\right)$. O pareamento foi realizado de acordo com características clínicas (idade e diabetes) e angiográficas (diâmetro do vaso e extensão da lesão), sendo os pacientes, em sua maioria, provenientes de nossa experiência unicêntrica com o Randomized study with the Sirolimus-eluting Velocity Balloon Expandable Stent (RAVEL). O desenho do estudo pode ser observado na Figura 1.

Os critérios de inclusão foram lesão coronária de novo, única, com diâmetro de estenose de $50 \%$ a

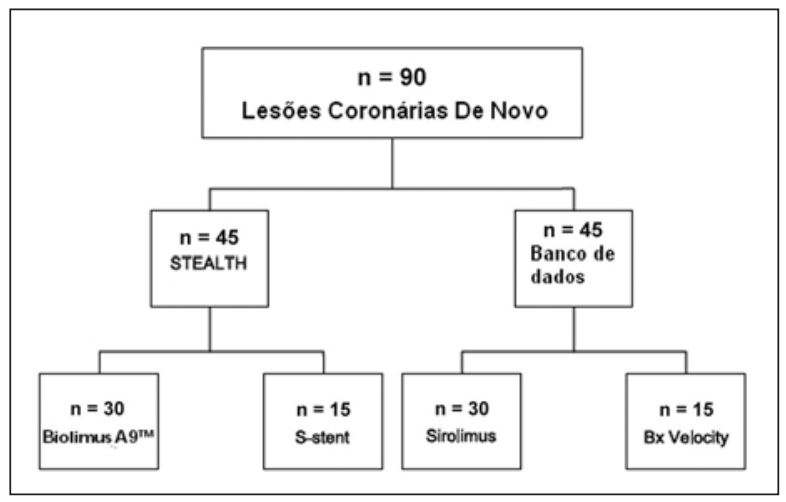

Figura 1 - Desenho do estudo.
$99 \%$, diâmetro de referência angiográfico de 2,5 mm a $4 \mathrm{~mm}$, e extensão da lesão inferior a $24 \mathrm{~mm}$. A prédilatação da lesão foi obrigatória. Pacientes apresentando lesão significante em tronco de artéria coronária esquerda (> 50\%), oclusão crônica total, fluxo TIMI inferior a 2, bifurcações envolvendo ramo secundário significante (diâmetro $>2 \mathrm{~mm}$ ), lesões ostiais, choque cardiogênico, fração de ejeção do ventrículo esquerdo inferior a 30\%, infarto agudo do miocárdio (IAM) transmural há menos de uma semana ou necessitando implante de múltiplos stents foram excluídos do estudo. Todos os pacientes assinaram o documento de consentimento informado, e o protocolo do estudo foi aprovado pela comissão de ética da instituição.

IAM foi definido como novas ondas Q patológicas em duas ou mais derivações contínuas ou elevação da creatina quinase $(\mathrm{CK})$ ou sua isoenzima $M B(C K-M B)>$ 3 vezes o valor superior da normalidade durante a hospitalização ou $>2$ vezes o limite superior da normalidade após a mesma. CK e CK-MB foram dosadas antes do procedimento, depois de 8-12 horas e de 1824 horas, e então diariamente até a alta hospitalar.

\section{Farmacoterapia adjuvante}

Todos os pacientes receberam farmacoterapia adjuvante, sendo pré-tratados com aspirina, em caso de não fazerem uso prévio (325 mg por via oral 24 horas antes do procedimento) e tienopiridínicos em dose de ataque (ticlopidina $500 \mathrm{mg}$ por via oral ou clopidogrel $300 \mathrm{mg}$ por via oral). Durante o procedimento, os pacientes receberam heparina não-fracionada intravenosa $(100 \mathrm{UI} / \mathrm{kg})$ com o objetivo de manter o tempo de coagulação ativado entre 250 e 350 segundos. Todos os pacientes receberam clopidogrel ou ticlopidina, por um período que variou de dois a seis meses após o implante de stents, e aspirina, continuamente. Os inibidores de glicoproteína foram utilizados a critério dos operadores.

\section{Aquisição das imagens}

A angiografia pós-procedimento foi realizada em pelo menos duas projeções ortogonais. As mesmas projeções ortogonais foram repetidas aos seis e 12 meses de seguimento. Realizamos, então, a aquisição de imagens dos stents implantados utilizando transdutor de elemento único, rotacional, com freqüência de 40 $\mathrm{MHz}$ (ClearView, Boston Scientific Corp., Nattick, Estados Unidos), sendo realizado recuo motorizado à velocidade de $0,5 \mathrm{~mm} / \mathrm{s}$. As imagens adquiridas foram armazenadas em fitas S-VHS para posterior reconstrução tridimensional.

\section{Angiografia coronária quantitativa}

Dois investigadores independentes, sem conhecimento do tipo de stent empregado em cada grupo, realizaram análise coronária quantitativa utilizando sistema de detecção automática de bordas (MEDIS Medical 
Imaging System, Leiden, Holanda). A ponta do cateter repleta de contraste foi utilizada para calibração. O diâmetro luminal mínimo pré e pós-procedimento, o diâmetro de referência e o diâmetro do balão em insuflação máxima foram mensurados.

\section{Análise volumétrica por ultra-som intracoronário}

A reconstrução tridimensional das imagens de ultrasom intracoronário foi realizada por meio do programa EchoPlaque 2 (Indec Systems Inc., Mountain View, Estados Unidos), por dois investigadores independentes e cegos para o tratamento instituído. Os segmentos referentes aos stents foram medidos a cada milímetro por planimetria computadorizada. As áreas referentes a stent, lúmen e hiperplasia neo-intimal (área do stent - área do lúmen) foram medidas no segmento analisado, e os volumes foram calculados pela regra de Simpson $^{6}$. O porcentual de obstrução volumétrica (\%OV) intra-stent foi calculado como a relação entre o volume de hiperplasia e o volume do stent X 100 e o índice de hiperplasia intimal, como a relação entre o volume absoluto de hiperplasia e a extensão do stent ${ }^{7}$. Aposição incompleta do stent foi definida como separação de uma ou mais hastes do stent da parede do vaso com presença de fluxo entre o stent e o vaso.

\section{Análise estatística}

A análise estatística foi realizada com o programa StatView 5.0.1 (SAS Institute, Cary, Estados Unidos). As variáveis numéricas foram expressas como média \pm desvio padrão e as categóricas, como números (n) e porcentagens $(\%)$. O teste do qui-quadrado e o teste exato de Fisher foram usados para variáveis categóricas; o teste $t$ de Student bicaudal foi utilizado nas comparações de variáveis contínuas com distribuição normal. Nas situações em que o conjunto de dados não passou nos testes de normalidade, foram utilizados testes não-paramétricos, como Mann-Whitney (para comparação entre dois grupos) e Kruskal-Wallis (para comparação de três ou mais grupos). De acordo com estudos previamente publicados envolvendo stents farmacológicos, o cálculo da amostra necessária para nosso estudo baseou-se na hipótese de porcentual de obstrução intra-stent em torno de $5 \%$ para os grupos com stents eluidores de medicamentos, e de ao menos $10 \%$ para os grupos dos stents convencionais, sendo uma diferença de 5\% no volume de obstrução entre os stents eluidores de medicamentos considerada clinicamente significativa para um valor de p-bicaudal de 0,05 e poder de 95\%. Valores de probabilidade $<0,05$ foram considerados estatisticamente significativos.

\section{RESULTADOS}

As características clínicas e angiográficas foram similares entre os grupos, com exceção de uma porcentagem significativamente maior de mulheres e de lesões do tipo C no grupo de stents eluidores de BA9
(Tabela 1). Não houve incidência de acidente vascular cerebral periprocedimento ou de sangramentos maiores ou menores para toda a amostra. Um (3,3\%) paciente no grupo de stents eluidores de BA9 apresentou IAM, porém não relacionado ao vaso-alvo, e outro $(3,3 \%)$ indivíduo do mesmo grupo apresentou reestenose com necessidade de revascularização do vaso-alvo. Um $(6,6 \%)$ paciente no grupo do sirolimus controle apresentou reestenose com necessidade de revascularização do vaso-alvo. Não foram observados casos de trombose aguda, subaguda ou tardia (até seis meses) em nenhum grupo. Não houve diferença estatisticamente significante entre os quatro grupos em relação a eventos cardíacos maiores (Tabela 2).

\section{Angiografia coronária quantitativa}

A análise angiográfica quantitativa foi realizada em todos os 90 (100\%) pacientes (Tabela 3). Diâmetro de referência do vaso pré-procedimento, ganho luminal agudo e extensão da lesão-alvo foram semelhantes entre os grupos. A perda tardia angiográfica foi significativamente maior nos grupos controle em comparação aos grupos dos stents eluidores de medicamentos (Figura 2). Entretanto, não houve diferença significativa na perda tardia angiográfica aos seis meses no grupo de stents eluidores de BA9 em comparação ao de stents eluidores de sirolimus. Não foi observado nenhum episódio de reestenose oclusiva nos quatro grupos.

\section{Análise volumétrica pelo ultra-som intracoronário}

A análise volumétrica intra-stent pelo ultra-som intracoronário aos seis meses foi realizada em todos os pacientes incluídos nesta análise, e está descrita na Tabela 4. O volume do stent e do lúmen foi significativamente menor no grupo do stent Bx-Velocity ${ }^{\mathrm{TM}}$ em comparação a ambos os grupos de stents farmacológicos. Entretanto, não houve diferença quanto à extensão dos stents entre os quatro grupos. De maneira semelhante aos resultados angiográficos, o porcentual de obstrução volumétrica foi significativamente inferior no grupo de stents eluidores de BA9 em comparação ao grupo controle, o mesmo sendo observado na comparação entre o grupo de stents eluidores de sirolimus com o respectivo grupo controle (Figura 3). O mesmo padrão de resultados foi observado na comparação do índice de hiperplasia intimal entre os grupos (Figura 4). Não houve diferença significativa no porcentual de obstrução volumétrica intra-stent na comparação direta do grupo de stents eluidores de BA9 com o grupo de stents eluidores de sirolimus (Figura 5). Não foi observado nenhum caso de aposição incompleta nos quatro grupos analisados.

\section{DISCUSSÃO}

O sirolimus tem propriedades macrolídeas e antifúngicas, exercendo potente efeito citostático nas cé- 
TABELA 1

Características clínicas e angiográficas

\begin{tabular}{|c|c|c|c|c|}
\hline & $\begin{array}{l}\text { Biolimus A9 } \\
\quad(n=30)\end{array}$ & $\begin{array}{l}\text { Biolimus controle } \\
\qquad(\mathrm{n}=15)\end{array}$ & $\begin{array}{l}\text { Sirolimus } \\
(\mathbf{n}=30)\end{array}$ & $\begin{array}{l}\text { Sirolimus controle } \\
\qquad(\mathrm{n}=15)\end{array}$ \\
\hline Idade & $58,3 \pm 9,1$ & $57,2 \pm 7,5$ & $59,4 \pm 9,8$ & $57,5 \pm 8,2$ \\
\hline Homens, n (\%) & $15(50)^{*}$ & $11(73)$ & $25(83)$ & $11(73)$ \\
\hline Diabetes, n (\%) & $6(20)$ & $2(13)$ & $10(33)$ & $5(33)$ \\
\hline Hipertensão, n (\%) & $24(80)$ & $14(93)$ & $24(80)$ & $10(67)$ \\
\hline Fumantes, n (\%) & $16(53)$ & $12(80)$ & $14(47)$ & $7(47)$ \\
\hline Dislipidemia, n (\%) & $19(63)$ & $5(33)$ & $16(53)$ & $11(73)$ \\
\hline IAM prévio, n (\%) & $15(50)$ & $9(60)$ & $10(33)$ & $7(47)$ \\
\hline ATC prévia, n (\%) & $2(7)$ & $0(0)$ & $3(10)$ & $3(20)$ \\
\hline CRM prévia, n (\%) & $4(13)$ & $1(7)$ & $1(3)$ & $0(0)$ \\
\hline ICC, n (\%) & $0(0)$ & $0(0)$ & $0(0)$ & $1(7)$ \\
\hline Angina estável, n (\%) & $21(70)$ & $9(60)$ & $27(90)$ & $11(73)$ \\
\hline \multicolumn{5}{|l|}{ Tipo de lesão ${ }^{+}$} \\
\hline A, n (\%) & $2(7)$ & $1(7)$ & $11(37)$ & $6(40)$ \\
\hline $\mathrm{B} 1, \mathrm{n}(\%)$ & $16(53)$ & $9(60)$ & $14(47)$ & $7(47)$ \\
\hline B2, n (\%) & $8(27)$ & $5(33)$ & $5(17)$ & $2(13)$ \\
\hline C, n (\%) & $4(13)^{\#}$ & $0(0)$ & $0(0)$ & $0(0)$ \\
\hline \multicolumn{5}{|l|}{ Vaso tratado } \\
\hline $\mathrm{DA}, \mathrm{n}(\%)$ & $10(33)$ & $5(33)$ & $12(40)$ & $10(67)$ \\
\hline CX, n (\%) & $8(27)$ & $5(33)$ & $5(17)$ & $1(7)$ \\
\hline $\mathrm{CD}, \mathrm{n}(\%)$ & $12(40)$ & $5(33)$ & $13(43)$ & $4(27)$ \\
\hline
\end{tabular}

+ Classificação do American College of Cardiology/American Heart Association. * $\mathrm{p}=0,04 ;$ * $\mathrm{p}=0,02$. Demais $\mathrm{p}=\mathrm{NS}$. $\mathrm{n}=$ número de pacientes; IAM = infarto agudo do miocárdio; $\mathrm{ATC}=$ angioplastia transluminal coronária; $\mathrm{CRM}=$ cirurgia de revascularização miocárdica; ICC = insuficiência cardíaca congestiva; $\mathrm{DA}=$ artéria descendente anterior; $\mathrm{CX}=$ artéria circunflexa; $\mathrm{CD}=$ artéria coronária direita; NS = não-significante.

TABELA 2

Seguimento clínico aos seis meses

\begin{tabular}{lcccc}
\hline & $\begin{array}{c}\text { Biolimus A9 } \\
(\mathbf{n}=\mathbf{3 0})\end{array}$ & $\begin{array}{c}\text { Biolimus controle } \\
(\mathbf{n}=\mathbf{1 5})\end{array}$ & $\begin{array}{c}\text { Sirolimus } \\
(\mathbf{n}=\mathbf{3 0})\end{array}$ & $\begin{array}{c}\text { Sirolimus controle } \\
(\mathbf{n}=\mathbf{1 5})\end{array}$ \\
\hline Eventos cardíacos maiores, n (\%) & $2(6,7)$ & $0(0)$ & $0(0)$ & $2(6,7)$ \\
Óbito, n (\%) & $0(0)$ & $0(0)$ & $0(0)$ & $0(0)$ \\
IAM, n (\%) & $1(3,3)^{*}$ & $0(0)$ & $0(0)$ & $0(0)$ \\
AVC, n (\%) & $0(0)$ & $0(0)$ & $0(0)$ & $0(0)$ \\
RVA - ATC, n (\%) & $1(3,3)$ & $0(0)$ & $0(0)$ & $2(6,7)$ \\
RVA - CRM, n (\%) & $0(0)$ & $0(0)$ & $0(0)$ & 0 \\
\hline
\end{tabular}

* Não relacionado ao vaso-alvo. Todos $\mathrm{p}=\mathrm{NS}$. $\mathrm{n}=$ número de pacientes; IAM = infarto agudo do miocárdio; AVC = acidente vascular cerebral; RVA = revascularização do vaso-alvo; ATC = angioplastia transluminal coronária; CRM = cirurgía de revascularização do miocárdio; NS = não-significante.

lulas musculares lisas do vaso ${ }^{8}$. Os stents eluidores de sirolimus apresentam dramática redução de $90 \%$ nas taxas de reestenose, reduzindo assim significativamente a necessidade de eventos cardíacos maiores, quando comparados ao grupo controle $e^{9,10}$. Essa redução da incidência de eventos cardíacos maiores se deve pri- mariamente à diminuição da necessidade de nova revascularização da lesão-alvo, efeito observado mesmo em situações clínico-angiográficas de alta complexida$\mathrm{de}^{11}$. O BA9, um dos fármacos pertencentes à família do sirolimus, recentemente descoberto, possui propriedades antiproliferativas similares, exercidas por meio 
TABELA 3

Angiografia coronária quantitativa

\begin{tabular}{|c|c|c|c|c|c|}
\hline & & $\begin{array}{c}\text { Biolimus A9 } \\
(\mathbf{n}=30)\end{array}$ & $\begin{array}{l}\text { Biolimus controle } \\
\quad(n=15)\end{array}$ & $\begin{array}{l}\text { Sirolimus } \\
(\mathbf{n}=30)\end{array}$ & $\begin{array}{l}\text { Sirolimus controle } \\
\qquad(\mathrm{n}=15)\end{array}$ \\
\hline \multirow[t]{3}{*}{ Procedimento } & Diâmetro de referência & $2,95 \pm 0,40^{+}$ & $2,97 \pm 0,43^{+}$ & $2,85 \pm 0,42^{+}$ & $3,15 \pm 0,25^{+}$ \\
\hline & Extensão da lesão & $14,5 \pm 4,22^{+}$ & $12,75 \pm 3,82^{+}$ & $12,86 \pm 3,11^{+}$ & $13,01 \pm 2,67^{+}$ \\
\hline & Ganho agudo & $2,14 \pm 0,35^{+}$ & $2,15 \pm 0,32^{+}$ & $1,91 \pm 0,40^{+}$ & $1,97 \pm 0,31^{+}$ \\
\hline \multirow[t]{3}{*}{ Seis meses } & Perda tardia & $0,24 \pm 0,39^{\phi} *$ & $0,71 \pm 0,47^{\phi}$ & $0,15 \pm 0,38^{\phi *}$ & $0,77 \pm 0,56^{\phi}$ \\
\hline & Diâmetro de estenose & $13,6 \pm 10,5^{\phi *}$ & $22,4 \pm 8,9^{\phi}$ & $11,95 \pm 8,6^{\phi *}$ & $34,13 \pm 27,1^{\phi}$ \\
\hline & Reestenose binária & $1(3,3 \%)$ & $0(0,0 \%)$ & $1(3,3 \%)$ & $1(6,7 \%)$ \\
\hline
\end{tabular}

TABELA 4

Análise volumétrica pelo ultra-som intracoronário aos seis meses

\begin{tabular}{|c|c|c|c|c|}
\hline & $\begin{array}{l}\text { Biolimus A9 } \\
\quad(n=30)\end{array}$ & $\begin{array}{l}\text { Biolimus controle } \\
\qquad(n=15)\end{array}$ & $\begin{array}{l}\text { Sirolimus } \\
(n=30)\end{array}$ & $\begin{array}{l}\text { Sirolimus controle } \\
\qquad(\mathrm{n}=15)\end{array}$ \\
\hline Volume do stent $\left(\mathrm{mm}^{3}\right)$ & $162,55 \pm 80,2^{+}$ & $167,81 \pm 65,2^{+}$ & $118,8 \pm 24,5^{+}$ & $130,80 \pm 30,3^{+}$ \\
\hline Volume do lúmen $\left(\mathrm{mm}^{3}\right)$ & $159,35 \pm 58,2^{+}$ & $139,23 \pm 54,1^{+}$ & $114,05 \pm 26,2^{+}$ & $93,80 \pm 27,0^{+}$ \\
\hline Extensão do stent (mm) & $19,26 \pm 6,04$ & $18,13 \pm 5,42$ & $18,04 \pm 0,66$ & $17,92 \pm 0,54$ \\
\hline $\begin{array}{l}\text { Índice de hiperplasia } \\
\text { intimal }\left(\mathrm{mm}^{3} / \mathrm{mm}\right)\end{array}$ & $0,19 \pm 0,08^{\phi *}$ & $2,71 \pm 0,50^{\phi}$ & $0,18 \pm 0,06^{\phi *}$ & $2,02 \pm 0,23^{\phi}$ \\
\hline $\begin{array}{l}\text { Porcentual de obstrução } \\
\text { volumétrica intra-stent (\%) }\end{array}$ & $2,23 \pm 0,77^{\phi} *$ & $19,92 \pm 4,65^{\phi}$ & $3,30 \pm 1,11^{\phi *}$ & $33,5 \pm 3,10^{\phi}$ \\
\hline
\end{tabular}

${ }^{+} \mathrm{p}=<0,05 ;{ }^{\dagger} \mathrm{p}<0,001$ para stents farmacológicos vs. controles; ${ }^{*} \mathrm{p}=\mathrm{NS}$ para biolimus A9 vs. sirolimus. $\mathrm{n}=\mathrm{número}$ de pacientes; NS = não-significante.

do bloqueio da fase G1-S do ciclo celular. Os resultados angiográficos e de ultra-som intracoronário do estudo STEALTH I, desenhado para avaliar a eficácia e a segurança dos stents eluidores de BA9, foram reportados recentemente, evidenciando alta efetividade, associada a baixa incidência de eventos cardíacos maiores $^{4,5}$.

As diferenças entre a molécula do BA9 e do sirolimus são primariamente estruturais, mas conferem perfil mais lipofílico ao BA9, que, portanto, teoricamente, possuiria eluição mais rápida. Em relação à plataforma, o $\mathrm{Bx}$ $V_{\text {elocity }}{ }^{\mathrm{M}}$ tem desenho tubular, de células fechadas, enquanto o S-stent ${ }^{\mathrm{TM}}$ possui um desenho em quadratura, mas a diferença mais marcante entre esses dois dispositivos está relacionada ao polímero empregado. O stent eluidor de sirolimus emprega um polímero permanente, que permanece na parede do vaso, mesmo após a eluição do fármaco. Evidências histopatológicas recentes têm atribuído aos polímeros duráveis reações de hipersensibilidade local, com infiltração eosinofílica resultando em maior potencial trombótico, além de possível papel na gênese da malaposição tardia dos stents ${ }^{12,13}$. Este último fenômeno, definido como a se- paração de uma ou mais hastes do stent da parede do vaso observada somente no seguimento $\operatorname{tardio}^{14}$, foi observado no seguimento ultra-sonográfico da maioria dos grandes estudos randomizados com stent eluidor de sirolimus ${ }^{15,16}$, porém nunca observado em stents farmacológicos que utilizaram um polímero bioabsorvível (ácido polilático) como polímero carreador ${ }^{17,18}$. O stent eluidor de BA9 também utiliza esse mesmo polímero, sendo liberado juntamente com o fármaco na parede do vaso, evitando o contato prolongado com o mesmo após o período de eluição e, portanto, evitando potencial efeito deletério na parede do vaso ao longo do tempo.

A análise volumétrica pelo ultra-som intracoronário é um método de quantificação da proliferação neointimal, que permite diferenciar, com maior acurácia, o desempenho de dois stents farmacológicos altamente efetivos. Os parâmetros de hiperplasia neo-intimal, como o porcentual de obstrução volumétrica e o índice de proliferação neo-intimal permitindo a detecção de diferença mínimas na proliferação neo-intimal, possuem boa correlação com a perda tardia angiográfica $^{19,20}$. Apesar de os volumes finais dos stents não 


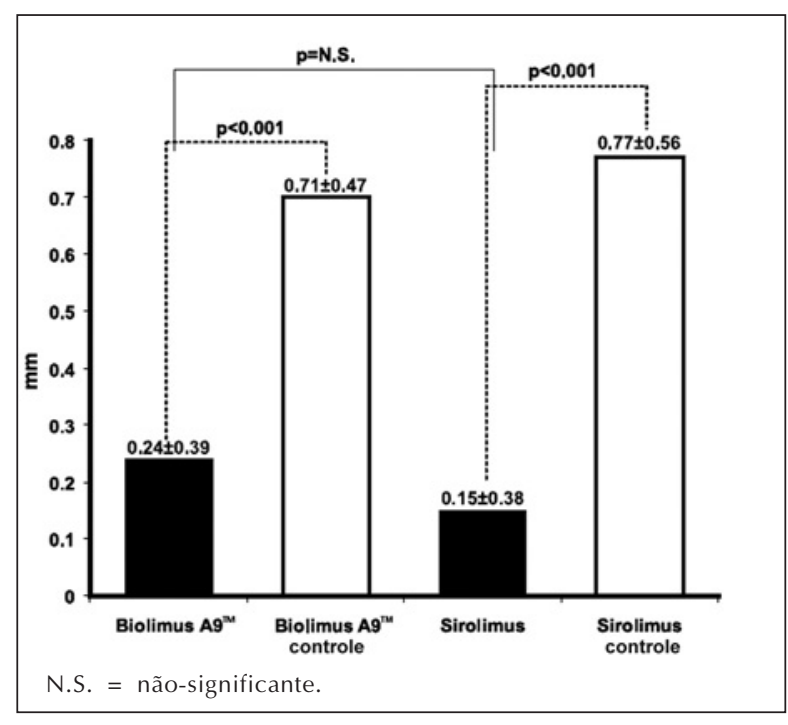

Figura 2 - Perda tardia angiográfica, aos seis meses de seguimento.

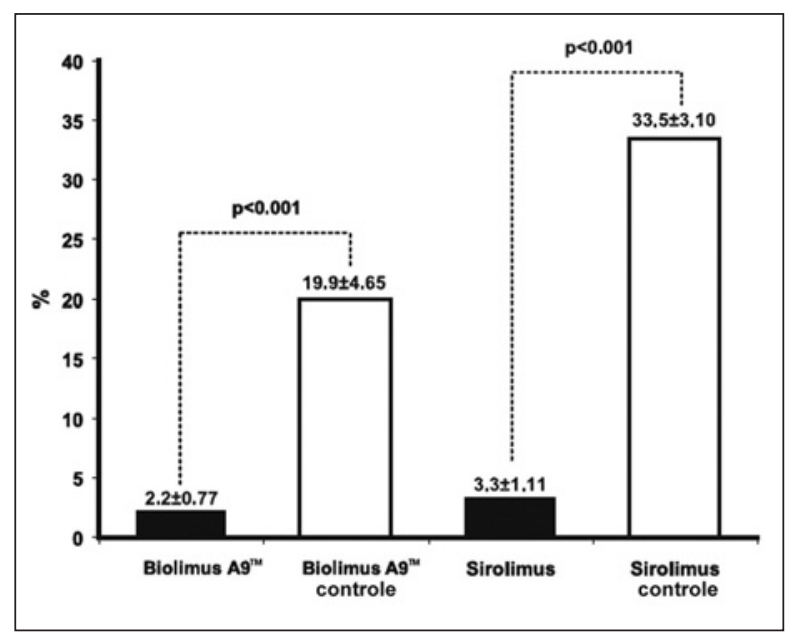

Figura 3 - Comparação do porcentual de obstrução volumétrica intra-stent (\%) nos pacientes submetidos a implante de stents farmacológicos vs. controles, aos seis meses de seguimento.

serem similares entre os grupos em nosso estudo, os parâmetros relativos de quantificação da hiperplasia utilizados são naturalmente corrigidos para variações no volume das endopróteses.

\section{Limitações do estudo}

A comparação entre dois stents farmacológicos realizada a partir do pareamento com um grupo controle histórico submete esta análise às potenciais limitações de um estudo não-randomizado. O período de seguimento de seis meses ainda não nos permite acessar a eficácia e a segurança tardia de ambos os dispositivos. A amostra limitada deste estudo impede comparação adequada dos stents eluidores de BA9 com o grupo controle em relação a desfechos clínicos ou

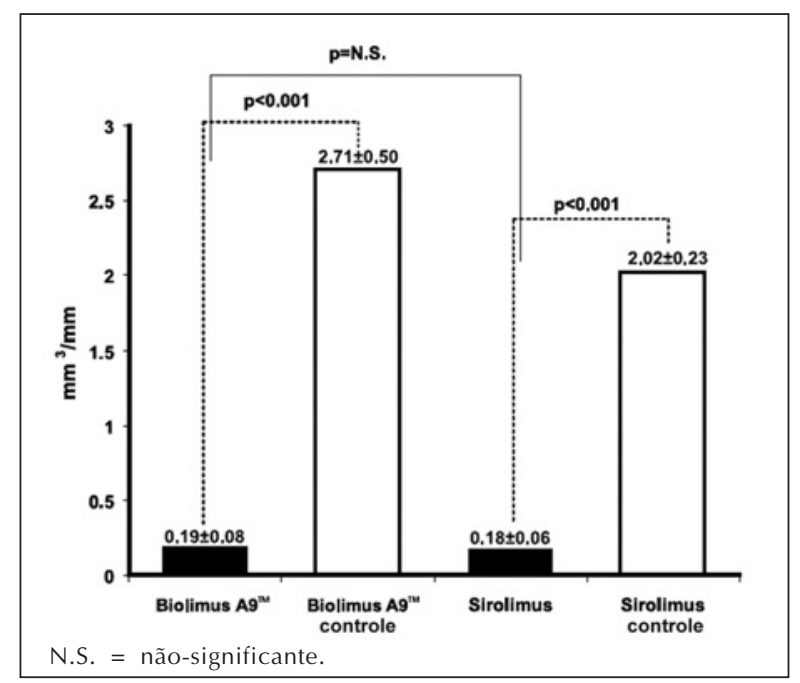

Figura 4 - Comparação do índice de hiperplasia intimal $\left(\mathrm{mm}^{3} / \mathrm{mm}\right)$ entre os grupos, aos seis meses.

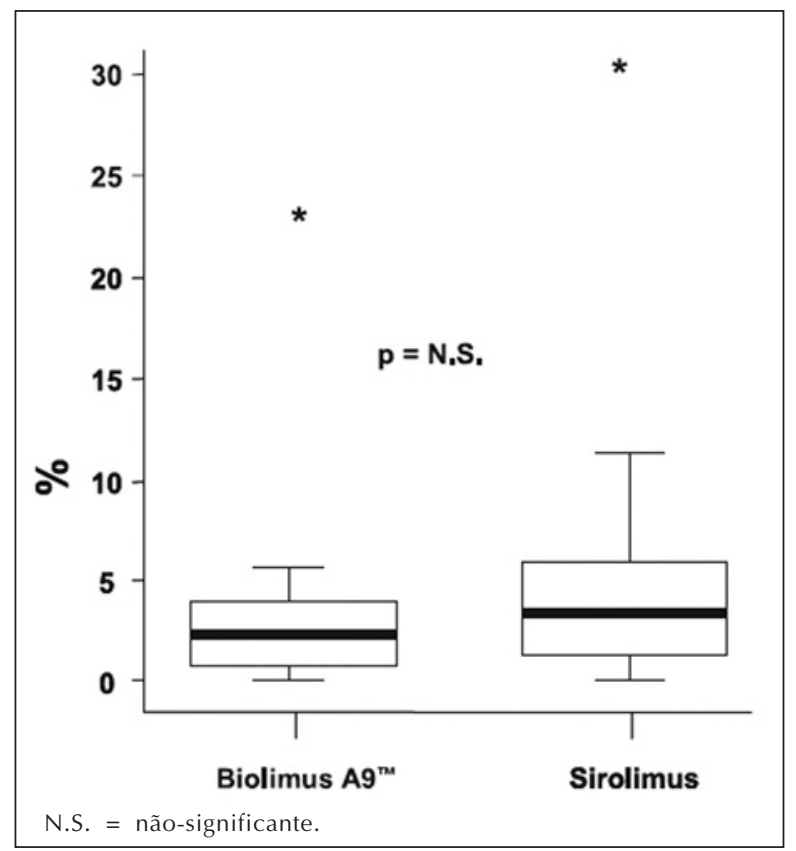

Figura 5 - Análise comparativa direta do porcentual de obstrução volumétrica intra-stent (\%), aos seis meses, entre os grupos que receberam implante de stents eluidores de biolimus A9 e de sirolimus.

reestenose binária. Um estudo multicêntrico, randomizado, controlado, com período maior de seguimento e incluindo casos de maior complexidade é, portanto, necessário para a comparação definitiva entre esses dois stents farmacológicos altamente eficazes.

\section{CONCLUSÃO}

Os stents eluidores de sirolimus e de BA9 apresentam potente e similar efeito na inibição da proliferação 
neo-intimal em pacientes submetidos a tratamento percutâneo de lesões coronárias, em cenário de baixa complexidade tanto clínica como angiográfica, ambos apresentando desempenho significativamente superior, se comparados aos respectivos grupos controle.

\section{REFERÊNCIAS BIBLIOGRÁFICAS}

1. Morice MC, Serruys PW, Sousa JE, Fajadet J, Ban Hayashi $E$, Perin $M$, et al. A randomized comparison of a sirolimuseluting-stent with a standard stent for coronary revascularization. N Engl J Med. 2002;346(23):1773-80.

2. Moses JW, Leon MB, Popma JJ, Fitzgerald PJ, Holmes DR, $\mathrm{O}^{\prime}$ Shaughnessy C, et al. Sirolimus-eluting stents versus standard stents in patients with stenosis in a native coronary artery. $N$ Engl J Med. 2003;349(14):1315-23.

3. Kar S, Aragon J, Paresky A, Tio F, Shulze J, Betts R, et al. 1139-52 Biolimus A9: a new generation rapamycin analogue inhibits intimal hyperplasia in a porcine model. J Am Coll Cardiol. 2004;43:A84.

4. Costa RA, Lansky AJ, Abizaid A, Mueller R, Tsuchiya Y, Mori K, et al. Angiographic results of the first human experience with the Biolimus A9 drug-eluting stent for de novo coronary lesions. Am J Cardiol. 2006;98(4):443-6.

5. Missel E, Beraldo P, Abizaid A, Mattos LAP, Feres F, Staico $R$, et al. Inibição da proliferação neointimal após o implante de stents eluidores de Biolimus A9: análise volumétrica tardia com ultra-som intracoronário. Rev Bras Cardiol Invas. $2007 ; 15(3): 234-9$

6. Mintz GS, Nissen SE, Anderson WD, Bailey SR, Erbel R, Fitzgerald PJ, et al. American College of Cardiology Clinical Expert Consensus Document on Standards for Acquisition, Measurement and Reporting of Intravascular Ultrasound Studies (IVUS). A report of the American College of Cardiology Task Force on Clinical Expert Consensus Documents. J Am Coll Cardiol. 2001;37(5):1478-92.

7. Mehran R, Mintz GS, Hong MK, Tio FO, Bramwell O, Brahimi $A$, et al. Validation of the in vivo intravascular ultrasound measurement of in-stent neointimal hyperplasia volumes. J Am Coll Cardiol. 1998;32(3):794-9.

8. Poon M, Marx SO, Gallo R, Badimon J, Taubman MB, Marks AR. Rapamycin inhibits vascular smooth muscle cell migration. J Clin Invest. 1996;98(10):2277-83.

9. Sawhney N, Moses JW, Leon MB, Kuntz RE, Popma JJ, Bachinsky W, et al. Treatment of left anterior descending coronary artery disease with sirolimus-eluting stents. Circulation. 2004;110(4):374-9.

10. Rensing BJ, Vos J, Smits PC, Foley DP, van den Brand MJ, van der Giessen WJ, et al. Coronary restenosis elimination with a sirolimus eluting stent: first European human experience with 6-month angiographic and intravascular ultrasonic follow-up. Eur Heart J. 2001;22(22):2125-30.

11. Valgimigli M, Percoco G, Malagutti P, Campo G, Ferrari F, Barbieri D, et al. Tirofiban and sirolimus-eluting stent vs abciximab and bare-metal stent for acute myocardial infarction: a randomized trial. JAMA. 2005;293(17):2109-17.

12. Virmani R, Guagliumi G, Farb A, Musumeci G, Grieco N, Motta $\mathrm{T}$, et al. Localized hypersensitivity and late coronary thrombosis secondary to a sirolimus-eluting stent: should we be cautious? Circulation. 2004;109(6):701-5.

13. Joner M, Finn AV, Farb A, Mont EK, Kolodgie FD, Ladich E, et al. Pathology of drug-eluting stents in humans: delayed healing and late thrombotic risk. J Am Coll Cardiol. 2006;48(1):193-202.

14. Serruys PW, Degertekin M, Tanabe K, Abizaid A, Sousa JE, Colombo A, et al. Intravascular ultrasound findings in the multicenter, randomized, double-blind RAVEL (RAndomized study with the sirolimus-eluting VElocity balloonexpandable stent in the treatment of patients with de novo native coronary artery Lesions) trial. Circulation. 2002;106(7): 798-803.

15. Degertekin M, Serruys PW, Tanabe K, Lee CH, Sousa JE, Colombo A, et al. Long-term follow-up of incomplete stent apposition in patients who received sirolimus-eluting stent for de novo coronary lesions: an intravascular ultrasound analysis. Circulation. 2003;108(22):2747-50.

16. Ako J, Morino Y, Honda Y, Hassan A, Sonoda S, Yock PG, et al. Late incomplete stent apposition after sirolimus-eluting stent implantation: a serial intravascular ultrasound analysis. J Am Coll Cardiol. 2005;46(6):1002-5.

17. Grube E, Sonoda S, Ikeno F, Honda Y, Kar S, Chan C, et al. Six- and twelve-month results from the first human experience using everolimus-eluting stents with bioabsorbable polymer. Circulation. 2004;109(18):2168-71.

18. Tsuchiya $\mathrm{Y}$, Lansky AJ, Costa RA, Mehran R, Pietras C, Shimada $Y$, et al. Effect of everolimus-eluting stents in different vessel sizes (from the pooled FUTURE I and II trials). Am J Cardiol. 2006;98(4):464-9.

19. von Birgelen C, Kutryk MJ, Serruys PW. Three-dimensional intravascular ultrasound analysis of coronary stent deployment and in-stent neointimal volume: current clinical practice and the concepts of TRAPIST, ERASER, and ITALICS. J Invasive Cardiol. 1998;10(1):17-26.

20. Prati F, Pawlowski T, Sommariva L, Labellarte A, Manzoli A, Boccanelli A, et al. Intravascular ultrasound and quantitative coronary angiography assessment of late in-stent restenosis: in vivo human correlation and methodological implications. Catheter Cardiovasc Interv. 2002;57(2):155-60. 